\title{
Racial Differences in Treatment and Outcomes Among Patients With Early Stage Bladder Cancer
}

Brent K. Hollenbeck, MD, MS ${ }^{1,2,3}$; Rodney L. Dunn, MS²; Zaojun Ye, MS²; John M. Hollingsworth, MD, MS ${ }^{2,4}$; Cheryl T. Lee, MD'; and John D. Birkmeyer, MD 3,5

BACKGROUND: Black patients are at greater of risk of death from bladder cancer than white patients. Potential explanations for this disparity include a more aggressive phenotype and delays in diagnosis resulting in higher stage disease. Alternatively, black patients may receive a lower quality of care, which may explain this difference. METHODS: Using Surveillance, Epidemiology, and End Results (SEER)-Medicare data for the years from 1992 through 2002, the authors identified patients with early stage bladder cancer. Multivariate models were fitted to measure relations between race and mortality, adjusting for differences in patients and treatment intensity. Next, shared-frailty proportional hazards models were fitted to evaluate whether the disparity was explained by differences in the quality of care provided. RESULTS: Compared with white patients $(n=14,271)$, black patients $(n=342)$ were more likely to undergo restaging resection ( $12 \%$ vs $6.5 \% ; P<.01$ ) and urine cytologic evaluation ( $36.8 \%$ vs $29.7 \%$; $P<.01$ ), yet they received fewer endoscopic evaluations ( 4 vs 5; $P<.01$ ). The use of aggressive therapies (cystectomy, systemic chemotherapy, radiation) was found to be similar among black patients and white patients ( $12 \%$ vs $10.2 \%$, respectively; $P=$ .31). Although black patients had a greater risk of death compared with white patients (hazards ratio [HR], 1.23; $95 \%$ confidence interval [95\% Cl], 1.07-1.42), this risk was attenuated only modestly after adjusting for differences in treatment intensity and provider effects (HR, 1.22; 95\% Cl, 1.06-1.42). CONCLUSIONS: Although differences in initial treatment were evident, they did not appear to be systematic and had unclear clinical significance. Whereas black patients are at greater risk of death, this disparity did not appear to be caused by differences in the intensity or quality of care provided. Cancer 2010;116:50-6. (C) 2010 American Cancer Society.

KEYWORDS: bladder cancer, disparities, quality of care, shared-frailty models.

Among those with bladder cancer, black patients have an approximately $70 \%$ greater risk of cancer-related death compared with white patients. ${ }^{1}$ Even among those with localized disease, black patients have significantly worse 10 -year disease-specific survival $(81 \%$ vs $88 \%) .^{2}$ Although the underpinnings for this disparity are not entirely clear, plausible explanations include a more aggressive cancer phenotype (ie, tumor biology), delays in diagnosis that result in higher stage disease at presentation, and a greater burden of comorbid diseases. Since early stage (ie, superficial or nonmuscle-invasive) bladder cancer traditionally is considered a nonlethal disease, comorbidity may be an important contributor to apparent cancer-related mortality because of difficulties in ascertaining the cause of death (ie, attribution bias). ${ }^{3}$

Alternatively, disparities in survival may be the end result of differences in the healthcare provided to black patients and white patients. On one hand, the disparity may reflect differential treatment by race. Among those with early stage lung cancer, the lower survival rate for black patients appears to be because patients in this group less frequently undergo surgery. ${ }^{4}$ With regard to early stage bladder cancer, differences in the use of surveillance (eg, endoscopy) and treatment (eg, intravesical therapy) may underlie the observed survival disparity. Conversely, survival differences may be attributable to the quality of care provided. Black patients who undergo radical cystectomy are nearly $70 \%$ more likely to die postoperatively compared with white patients, ${ }^{5}$ a finding that is largely a consequence of the quality of the hospital setting ${ }^{6}$ in which black patients more commonly receive their care (ie, low volume with limited access to potentially necessary health

Corresponding author: Brent K. Hollenbeck, MD, MS, Department of Urology, 1032B Michigan House, 2301 Commonwealth Boulevard, Ann Arbor, MI 48109-0330; Fax: (734) 232-2400; bhollen@umich.edu

${ }^{1}$ Division of Oncology, Department of Urology, University of Michigan, Ann Arbor, Michigan; ${ }^{2}$ Division of Health Services Research, University of Michigan, Ann Arbor, Michigan; ${ }^{3}$ The Michigan Surgical Collaborative for Outcomes Research and Evaluation, University of Michigan, Ann Arbor, Michigan; ${ }^{4}$ Robert Wood Johnson Clinical Scholars Program, University of Michigan, Ann Arbor, Michigan; ${ }^{5}$ Department of Surgery, University of Michigan, Ann Arbor, Michigan

DOI: 10.1002/cncr.24701, Received: February 2, 2009; Revised: April 8, 2009; Accepted: April 20, 2009, Published online October 28, 2009 in Wiley InterScience (www.interscience.wiley.com) 
services). In the setting of early stage bladder cancer, the physician, rather than the hospital, plays a principal role in determining treatment and outcomes. Because black patients and white patients generally receive their care by dissimilar physicians, ${ }^{7}$ differences in the quality of the bladder cancer care provided may explain the observed disparity in mortality.

For this reason, we undertook a study to better understand racial differences in the treatment of patients with early stage bladder cancer. Using national Surveillance, Epidemiology, and End Results (SEER)-Medicare data, we explored the extent to which disparities in mortality are explained by differences in the intensity and quality of the care provided.

\section{MATERIALS AND METHODS}

\section{Study Population}

We used the SEER-Medicare linked database for the years 1992 through 2002 to identify patients who were diagnosed with early stage bladder cancer. These files provide information on Medicare patients who are included in SEER, ${ }^{8}$ a collection of population-based registries of all incident cancers that comprised approximately $26 \%$ of the United States population by the end of the study period. ${ }^{9}$ For each Medicare patient in SEER, the SEERMedicare linked files contain 100\% of Medicare claims from the inpatient, outpatient, and national claims history files.

From these files, all fee-for-service Medicare patients ages 65 years to 99 years with incident cases of bladder cancer were identified by the appropriate code in SEER. We limited our study population to patients with early stage bladder cancer (noninvasive papillary carcinoma [Ta], carcinoma in situ [Tis], and tumor invading subepithelial connective tissue [T1] ${ }^{10}$ using the extent-of-disease codes provided by SEER. All patients were followed using Medicare claims through December 31, 2005.

\section{Characterization of Treatment}

We explored how patients with bladder cancer were managed using International Classification of Diseases, Ninth Revision (ICD-9) procedure and Healthcare Common Procedure Coding System (HCPCS) codes recorded in the Medicare files during the first 2 years after diagnosis. We focused on practices that were relevant to early stage bladder cancer care, including surveillance (endoscopic examination of the bladder, upper urinary tract evaluation, urinary studies, and imaging studies), treatment (intravesical therapy and repeat endoscopic resection within 60 days of the initial resection), and medical services (visits to the urologist and visits to other physicians).

To serve as a proxy for initial treatment intensity, we used early stage bladder cancer expenditures within the first 2 years after diagnosis. Briefly, expenditures were measured at the patient level and included all Medicare payments associated with a primary diagnosis of bladder cancer (ICD-9 codes: 188.x, bladder cancer; 233.7, carcinoma in situ of the bladder; and V105.4, personal history of bladder cancer). Expenditures related to major interventions (cystectomy, chemotherapy, and radiotherapy) were not included. All payments were price-adjusted to 2005 US dollars using the Medicare Economic Index and were standardized by region. ${ }^{11}$

Because an objective of our study was to evaluate the extent to which any observed disparity in mortality was attributable to the quality of care provided by the treating physician, it was necessary to assign each patient to a provider. To ascertain the physician who was primarily responsible for each patient's bladder cancer care, we first identified all bladder cancer-related procedures, as described by others, ${ }^{12}$ that were performed within a 2 year period after their diagnosis. Next, we allocated each patient to the provider with the majority of claims using the Unique Physician Identifier Number. Because it was necessary to obtain reliable estimates of physician's practice styles, we limited our study only to those physicians who had $\geq 10$ patients. Using this method, our final population was comprised of 14,613 patients who were treated by 656 providers.

\section{Outcomes}

Our primary outcome measure was mortality, which was assessed from January 1, 1992 through December 31, 2005 . Because of concerns regarding appropriately assigning the cause of death, ${ }^{3,13-16}$ we used all-cause mortality as our primary outcome. However, recognizing that the vast majority of patients with early stage bladder cancer are likely to die from competing causes, ${ }^{17}$ we also measured bladder cancer-specific mortality using the cause-of-death field available in SEER. We also assessed the use of a major intervention, as evidenced by the use of radical cystectomy, systemic chemotherapy, and radiotherapy, which could occur at any time during the study period. Furthermore, a composite variable was constructed to reflect the downstream use of any of these therapies. These secondary outcomes were identified by using appropriate ICD-9 and HCPCS codes in the inpatient, national claims history, and outpatient files. 


\section{Statistical Analysis}

For all of our analyses, our exposure was patient-level race (white, black) as measured by SEER. First, we examined differences in patient demographics and disease characteristics according to race. Then, we characterized the extent to which early stage bladder cancer care (surveillance, treatment, and medical services) varied by race. For all of these comparisons, statistical inference was made using chi-square or Kruskal-Wallis tests for categoric and continuous data, respectively.

For the purpose of understanding the correlation between race and mortality, we fitted Cox proportional hazards models that were adjusted for patient and disease characteristics, including patient age group (5-year intervals), sex, tumor grade (low, medium, high, and unknown), and tumor stage (Ta, Tis, $\mathrm{T} 1$, and $\mathrm{Ta} / \mathrm{T} 1$ unspecified). ${ }^{10}$ In addition, we adjusted for socioeconomic status using a composite measure assessed at the ZIP code level, as described by Diez-Roux et al. ${ }^{18}$ Patient comorbidities were identified by using healthcare encounters in the 12-month period preceding the bladder cancer diagnosis according to the well established methods described by Klabunde et al. ${ }^{19}$ Next, we evaluated the extent to which variation in the intensity of initial treatment for bladder cancer provided might explain differences in mortality by adjusting for patient-level treatment intensity. Finally, we explored whether the provider contributed to any observed disparities in survival by fitting a shared-frailty proportional hazards model, including a provider-level, random-effects term. ${ }^{20}$ This approach accounts for the correlation of mortality outcomes within a provider and for the heterogeneity between providers.

For the secondary outcomes (use of cystectomy, systemic chemotherapy, and/or radiotherapy), we fitted generalized estimating equations to evaluate the correlation between race and each patient-level outcome, adjusting for age, sex, comorbidity, socioeconomic status, tumor grade, and tumor stage. This approach allowed us to account for the potential correlation of our observations (ie, patients clustered within providers). ${ }^{21}$ We then used postestimation commands to predict adjusted percentages for the receipt of each intervention by race.

All analyses were performed using computerized software (SAS version 9.2 [SAS Inc, Cary, NC] Stata [version 10; Stata Corp., College Station, Tex]). All tests were 2-tailed, and the probability of Type 1 error was set at .05 . The study protocol was approved by the Institutional Review Board of the University of Michigan.
Table 1. Patient Characteristics by Race

\section{Characteristic}

No. of patients (\%)

Median treatment

intensity, 2005 US\$*

Age, $\%$

$65-69$ y

70-74 y

$75-79$ y

80-84 y

$\geq 85 \mathrm{y}$

Women, \%

Socioeconomic status, $\%$

Low

Medium

High

No. of comorbidities, \%

0

1

2

$\geq 3$

Tumor grade, \%

Low

Medium

High

Unknown

AJCC tumor stage, \%

$\mathrm{Ta}$

Tis

T1

Unspecified

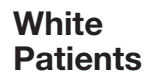

$14,271(97.7)$

$\$ 2778$

14.0
24.4
25.8
20.4
15.4
25.4


29.8
35.7
34.4

43.4

30.2

14.8

11.6

19.5

45.8

27.6

7.1

57.2

6.9

24.6

11.3
Black $P$ Patients

342 (2.3) $\$ 2768 \quad .61$

.57

13.2

24.9

29.5

19.0

13.5

32.7

.003

$<.001$

74.3

19.0

6.7

35.4

30.7

21.6

12.3

17.8

40.6

31.3

10.2

54.3

8.9

26.7

10.2
AJCC indicates American Joint Committee on Cancer; Tis, tumor in situ. * Represents expenditures median per capita expenditures for the first 2 years after diagnosis.

\section{RESULTS}

Black patients with early stage bladder cancer had a significantly lower median overall survival rate compared with white patients ( 4.4 years vs 6.5 years; log-rank $P<.001$ ). Table 1 illustrates differences in patient and disease characteristics according to race. It is interesting to note that black patients had lower socioeconomic status and higher levels of comorbidity ( $P<.01$ for both). However, disease severity at diagnosis, as measured by cancer grade and stage, did not vary by race.

The initial treatment of early stage bladder cancer care varied according to race (Table 2). Generally, black patients were followed more intensively with urine cytology $(0.80$ tests vs 0.71 tests; $P<.01)$. Moreover, black patients were nearly twice as likely to undergo restaging resection of their cancer compared with white patients $(12 \%$ vs $6.5 \% ; P<.01)$. In contrast, black patients had 
Table 2. Differences in Early Stage Bladder Cancer Care During the First 2 Years After Diagnosis

\begin{tabular}{|c|c|c|c|}
\hline Process of Care & $\begin{array}{l}\text { White } \\
\text { Patients }\end{array}$ & $\begin{array}{l}\text { Black } \\
\text { Patients }\end{array}$ & $\boldsymbol{P}$ \\
\hline \multicolumn{4}{|l|}{ Surveillance-related } \\
\hline $\begin{array}{l}\text { Endoscopic } \\
\text { surveillance, mean }\end{array}$ & 5.0 & 4.0 & $<.001$ \\
\hline $\begin{array}{l}\text { Any upper urinary } \\
\text { tract evaluation, } \% \dagger\end{array}$ & 24.9 & 25.7 & .70 \\
\hline $\begin{array}{l}\text { Radiographic imaging } \\
\text { studies, mean* }\end{array}$ & 0.67 & 0.65 & .94 \\
\hline Urinary cytology, mean* & 0.71 & 0.80 & .009 \\
\hline Any urine cytology, $\% \dagger$ & 29.7 & 36.8 & .006 \\
\hline Urinalysis, mean* & 4.5 & 3.4 & $<.001$ \\
\hline \multicolumn{4}{|l|}{ Treatment-related } \\
\hline $\begin{array}{l}\text { Intravesical therapy, } \\
\text { mean* }^{*}\end{array}$ & 3.5 & 3.3 & .65 \\
\hline $\begin{array}{l}\text { Induction courses } \dagger \\
\text { of intravesical therapy, mean* }\end{array}$ & 0.30 & 0.26 & .51 \\
\hline $\begin{array}{l}\text { Any induction } \\
\text { intravesical therapy, } \% \dagger\end{array}$ & 26.8 & 25.7 & .67 \\
\hline $\begin{array}{l}\text { Repeat endoscopic } \\
\text { resection, } \% \dagger\end{array}$ & 6.5 & 12.0 & $<.001$ \\
\hline \multicolumn{4}{|l|}{ Medical services } \\
\hline $\begin{array}{l}\text { Visits to the } \\
\text { urologist, mean* }\end{array}$ & 3.8 & 3.7 & .92 \\
\hline $\begin{array}{l}\text { Visits to the other } \\
\text { physicians, mean* }\end{array}$ & 21.7 & 25.5 & $<.001$ \\
\hline
\end{tabular}

${ }^{*}$ For continuous measures (eg, endoscopic surveillance), the mean represents the average number of the services performed for each patient. For example, on average, black patients underwent 4 endoscopic procedures, and white patients underwent 5 endoscopic procedures during the first 2 years after diagnosis.

$\dagger$ For categoric measures (eg, any upper tract evaluation), the percentage represents the fraction of patients receiving that service. For example, $25.7 \%$ of black patients and $24.9 \%$ of white patients had any upper tract imaging performed within the first 2 years after diagnosis.

$\ddagger$ An induction course represents at least 5 instillations within a 45-day period.

fewer endoscopic bladder cancer evaluations compared with white patients ( 4 studies vs 5 studies; $P<.01$ ). The use of intravesical therapy did not vary by race.

The overall use of downstream major interventions was similar among black patients and white patients (Fig. 1). Although black patients were more likely to receive radiotherapy $(5.6 \%$ vs $3.2 \% ; P=.02)$ and systemic chemotherapy $(8.1 \%$ vs $5.3 \% ; P=.04)$ compared with white patients, the use of radical cystectomy was similar between the 2 groups. On average, the use of any major intervention did not vary significantly by race, and $12 \%$ of black patients and $10.2 \%$ of white patients received treatment $(P=.31)$.

Black patients had a $23 \%$ greater risk of death compared with white patients after adjusting for differences in clinical characteristics, as illustrated in Table 3. This risk

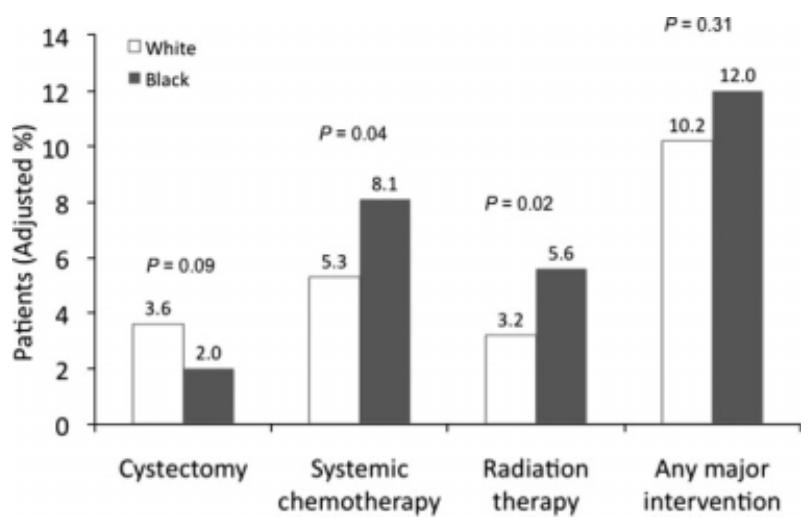

Figure 1. These charts illustrate the use of major interventions stratified by race and adjusted for age, sex, socioeconomic status, comorbidity, cancer grade, and cancer stage.

was attenuated only modestly after adjusting for differences in treatment intensity and the effect of the provider (adjusted hazards ratio, 1.22; 95\% confidence interval, 1.06-1.42). Similar modest attenuations were evident within stage strata and when using cancer-specific survival as the outcome.

\section{DISCUSSION}

Black patients who are diagnosed with early stage bladder cancer are at greater risk of death compared with white patients. However, this disparity did not appear to be caused by presentation with more severe disease, as measured by bladder cancer stage and grade. Not surprisingly, early stage bladder cancer care varied by race, although the majority of these differences had questionable clinical significance. Lacking context, these findings may suggest gaps in the quality of care as a principal source for this disparity. However, differences in initial treatment intensity and in the provider responsible for the bladder cancer care failed to account for virtually any of the excess mortality risk. Furthermore, the use of major medical interventions, including radical cystectomy, was similar among black and white patients.

Racial disparities in survival after a cancer diagnosis have been well described for a variety of malignancies, including bladder cancer. ${ }^{2,5,6}$ In 1 study, black patients had a nearly $70 \%$ greater risk of dying from bladder cancer compared with white patients. ${ }^{1}$ However, investigations into the underpinnings of this disparity largely have focused on delayed diagnosis ${ }^{2}$ or perioperative care after radical cystectomy, which traditionally has had higher operative mortality rates for blacks. ${ }^{5,6}$ In 1 study that used national data, black patients were $66 \%$ more likely to die 
Table 3. Effects of Treatment Intensity and Provider on Risk of Mortality

\begin{tabular}{|c|c|c|c|}
\hline Model/Category & $\begin{array}{l}\text { Adjusted* } \\
\text { HR }(95 \% \mathrm{Cl})\end{array}$ & $\begin{array}{l}\text { Adjusted } \dagger \\
\text { HR }(95 \% \mathrm{Cl})\end{array}$ & $\begin{array}{l}\text { Adjusted } \ddagger \\
\text { HR }(95 \% \mathrm{Cl})\end{array}$ \\
\hline \multicolumn{4}{|l|}{ All-cause mortality } \\
\hline \multicolumn{4}{|l|}{ All patients } \\
\hline White patients & 1.0 & 1.0 & 1.0 \\
\hline Black patients & $1.23(1.07-1.42)$ & $1.22(1.06-1.42)$ & $1.22(1.06-1.42)$ \\
\hline \multicolumn{4}{|c|}{ By AJCC tumor stage } \\
\hline \multicolumn{4}{|c|}{$\mathrm{Ta}$} \\
\hline White patients & 1.0 & 1.0 & 1.0 \\
\hline Black patients & $1.33(1.08-1.63)$ & $1.34(1.09-1.66)$ & $1.35(1.09-1.66)$ \\
\hline \multicolumn{4}{|l|}{$\mathrm{T} 1$} \\
\hline White patients & 1.0 & 1.0 & 1.0 \\
\hline Black patients & $1.41(1.10-1.83)$ & $1.40(1.09-1.81)$ & $1.40(1.08-1.81)$ \\
\hline \multicolumn{4}{|l|}{ Tis } \\
\hline White patients & 1.0 & 1.0 & 1.0 \\
\hline Black patients & $1.03(0.64-1.65)$ & $1.04(0.65-1.66)$ & $1.05(0.62-1.76)$ \\
\hline \multicolumn{4}{|c|}{ Cancer-specific mortality } \\
\hline \multicolumn{4}{|c|}{ All patients } \\
\hline White patients & 1.0 & 1.0 & 1.0 \\
\hline Black patients & $1.79(1.30-2.47)$ & $1.85(1.35-2.56)$ & $1.73(1.23-2.43)$ \\
\hline \multicolumn{4}{|c|}{ By AJCC tumor stage } \\
\hline \multicolumn{4}{|c|}{$\mathrm{Ta}$} \\
\hline White patients & 1.0 & 1.0 & 1.0 \\
\hline Black patients & $2.28(1.34-3.87)$ & $2.37(1.40-4.02)$ & $2.34(1.33-4.10)$ \\
\hline \multicolumn{4}{|l|}{$\mathrm{T} 1$} \\
\hline White patients & 1.0 & 1.0 & 1.0 \\
\hline Black patients & $1.79(1.11-2.88)$ & $1.83(1.14-2.95)$ & $1.86(1.12-3.09)$ \\
\hline \multicolumn{4}{|l|}{ Tis } \\
\hline White patients & 1.0 & 1.0 & 1.0 \\
\hline Black patients & $2.16(0.75-6.20)$ & $2.21(0.76-6.41)$ & $2.24(0.72-6.99)$ \\
\hline $\begin{array}{l}\text { HR indicates hazards rati } \\
\text { situ. } \\
\text { "Adjusted for age, sex, } \\
\text { was not included as a co } \\
\dagger \text { Adjusted for the above- } \\
\vdots \text { Adjusted for the above- }\end{array}$ & $\begin{array}{l}\text { confidence interval; } \\
\text { atus, comorbidity, gr }\end{array}$ & $\begin{array}{l}\text { nerican Joint Comm } \\
\text { tumor stage (note, fc } \\
\text { t intensity. }\end{array}$ & $\begin{array}{l}\text { ancer; Tis, tumor in } \\
\text { trata models, stage }\end{array}$ \\
\hline
\end{tabular}

perioperatively compared with white patients, even after adjusting for patient differences. ${ }^{5}$ For such high-risk procedures, it is believed generally that these disparities are a direct reflection of hospital quality and the finding that minorities generally seek care at lower quality hospitals. ${ }^{22}$ In fact, white patients and black patients tend to have similar mortality rates when they are treated at the same hospital, ${ }^{6}$ underscoring the importance of the setting and the provider to high-risk operations.

It is interesting to note that the current study illustrates that racial disparities in mortality are equally evident for those with early stage bladder cancer. Among patients who are diagnosed with bladder cancers in all stages, black patients are diagnosed with more advanced disease, ${ }^{23}$ a discrepancy that generally has been thought to underlie the observed survival differences. However, in the current study of patients with early stage disease, we did not observe a predilection for more aggressive phenotypes (eg, $\mathrm{T} 1$ bladder cancer) by race. That is, racial differences in survival in this population were not secondary to higher grade and higher stage bladder cancer. Furthermore, in contrast to the literature surrounding radical cystectomy, we observed no protective effect of the provider on survival differences. Indeed, our data indicate that the mortality disparity is persistent and equally robust even after adjusting for differences in the provider and the treatment intensity.

A principal limitation of our analysis relates to unmeasured patient differences that may confound correlations between race and mortality, an important consideration given the relatively small number of black patients in the study. In particular, black patients may have more aggressive bladder cancer and medical diseases that explain disparities in mortality risk. We addressed this well described limitation of observational data ${ }^{24,25}$ in 
several ways. First, we used a clinical registry to ascertain cancer stage and grade, which, arguably, are the most important determinants of death in the bladder cancer population. ${ }^{26,27}$ Second, we ascertained comorbid conditions using a well described methodology ${ }^{19}$ that incorporated data from both inpatient and outpatient claims. Because of entitlement issues, our comorbidity assessment (using 12 months of data before diagnosis) may underestimate the disease burden among patients aged 65 years, who would have more limited claims information. However, these patients had a median entitlement period of approximately 8 months (range, 45-365 days), so the effects of such underestimation most likely are limited. Furthermore, although more detailed measures of patients' health status may improve our ability to adjust according to risk, this would require a large, detailed clinical registry, which is not possible for practical reasons (eg, cost, sample size). Although we accounted for additional demographic differences using a composite measure for socioeconomic status, ${ }^{18}$ which is a well described predictor of long-term mortality, ${ }^{28}$ we recognize that race and class are complex constructs that cannot always be captured comprehensively in administrative data.

As with any observational study, there are additional limitations to consider. Because we used national SEERMedicare data, our findings may not be generalizable to patients aged $<65$ years. However, because nearly $75 \%$ of bladder cancers occur within the Medicare population, ${ }^{9}$ the extrapolation of our findings to a broader cohort appears to be reasonable. Finally, we acknowledge that race, as captured in SEER data, represents a constellation of constructs as described by others, ${ }^{29}$ including acculturation, education, socioeconomic class, and socialization. Future work should seek to disentangle this complicated web and evaluate these relations in other minority populations.

Compared with white patients who have early stage bladder cancer, black patients are at significantly greater risk of death. This disparity is not attributable to the diagnosis with more aggressive disease, the initial treatment intensity, or the quality of care provided by the urologist. Eliminating disparities in mortality for this chronic disease likely will require searching looking beyond the factors that pertain to healthcare delivery alone. Such factors, including behavior modification (eg, smoking cessation) and greater use of other preventive services, inevitably may lie upstream from the diagnosis of bladder cancer, imparting significant, but not insurmountable, challenges for future research.

\section{CONFLICT OF INTEREST DISCLOSURES}

Dr. Hollenbeck was supported by the American Cancer Society Pennsylvania Division-Dr. William and Rita Conrady Mentored Research Scholar Grant (MSRG-07-006-01-CPHPS), the American Urological Association Foundation, and Astellas Pharma US, Inc. Dr. Birkmeyer was supported by the National Cancer Institute (grants R01 CA098481-01 A1 and K05 CA115571-01 A2).

\section{REFERENCES}

1. Bach PB, Schrag D, Brawley OW, Galaznik A, Yakren S, Begg CB. Survival of blacks and whites after a cancer diagnosis. JAMA. 2002;287:2106-2113.

2. Lee CT, Dunn RL, Williams C, Underwood W, 3rd. Racial disparity in bladder cancer: trends in tumor presentation at diagnosis. J Urol. 2006;176:927-933.

3. Bach PB, Guadagnoli E, Schrag D, Schussler N, Warren JL. Patient demographic and socioeconomic characteristics in the SEER-Medicare database applications and limitations. Med Care. 2002;40(8 suppl):IV-19-IV-25.

4. Bach PB, Cramer LD, Warren JL, Begg CB. Racial differences in the treatment of early stage lung cancer. $N$ Engl $J$ Med. 1999;341:1198-1205.

5. Taub DA, Hollenbeck BK, Cooper KL, et al. Racial disparities in resource utilization for cystectomy. Urology. 2006; 67:288-293.

6. Lucas FL, Stukel TA, Morris AM, Siewers AE, Birkmeyer JD. Race and surgical mortality in the United States. Ann Surg. 2006;243:281-286.

7. Bach PB, Pham HH, Schrag D, Tate RC, Hargraves JL. Primary care physicians who treat blacks and whites. $N$ Engl J Med. 2004;351:575-584.

8. Warren JL, Klabunde CN, Schrag D, Bach PB, Riley GF. verview of the SEER-Medicare data: content, research applications, and generalizability to the United States elderly population. Med Care. 2002;40(8 suppl):IV-3-IV-18.

9. National Cancer Institute. Surveillance, Epidemiology, and End Results (SEER) Program. Available at http://seer. cancer.gov/. Accessed on February 1, 2009.

10. Greene FL, Page EL, Fleming ID, et al, eds. AJCC Cancer Staging Manual. 6th ed. New York, NY: Springer-Verlag; 2002.

11. Brown ML, Riley GF, Schussler N, Etzioni R. Estimating health care costs related to cancer treatment from SEERMedicare data. Med Care. 2002;40(8 suppl):IV-104-IV-117.

12. Schrag D, Hsieh LJ, Rabbani F, Bach PB, Herr H, Begg CB. Adherence to surveillance among patients with superficial bladder cancer. J Natl Cancer Inst. 2003;95:588597.

13. Hoel DG, Ron E, Carter R, Mabuchi K. Influence of death certificate errors on cancer mortality trends. J Natl Cancer Inst. 1993;85:1063-1068.

14. Feuer EJ, Merrill RM, Hankey BF. Cancer surveillance series: interpreting trends in prostate cancer-part II: cause of death misclassification and the recent rise and fall in prostate cancer mortality. J Natl Cancer Inst. 1999;91:1025-1032.

15. Penson DF, Albertsen PC, Nelson PS, Barry M, Stanford JL. Determining cause of death in prostate cancer: are death certificates valid? J Natl Cancer Inst. 2001;93:1822-1823.

16. Weinstock MA, Reynes JF. Validation of cause-of-death certification for outpatient cancers: the contrasting cases of melanoma and mycosis fungoides. Am J Epidemiol. 1998; 148:1184-1186. 
17. Herr HW. Tumor progression and survival of patients with high grade, noninvasive papillary (TaG3) bladder tumors: 15-year outcome. J Urol. 2000;163:60-61.

18. Diez Roux AV, Merkin SS, Arnett D, et al. Neighborhood of residence and incidence of coronary heart disease. $N$ Engl J Med. 2001;345:99-106.

19. Klabunde CN, Potosky AL, Legler JM, Warren JL. Development of a comorbidity index using physician claims data. J Clin Epidemiol. 2000;53:1258-1267.

20. Liu L, Wolfe RA, Huang X. Shared frailty models for recurrent events and a terminal event. Biometrics. 2004;60: 747-756.

21. Panageas KS, Schrag D, Riedel E, Bach PB, Begg CB. The effect of clustering of outcomes on the association of procedure volume and surgical outcomes. Ann Intern Med. 2003;139:658-665.

22. Liu JH, Zingmond DS, McGory ML, et al. Disparities in the utilization of high-volume hospitals for complex surgery. JAMA. 2006;296:1973-1980.

23. Virnig BA, Baxter NN, Habermann EB, Feldman RD, Bradley CJ. A matter of race: early versus late-stage cancer diagnosis. Health Aff. 2009;28:160-168.
24. Stukel TA, Fisher ES, Wennberg DE, Alter DA, Gottlieb DJ, Vermeulen MJ. Analysis of observational studies in the presence of treatment selection bias: effects of invasive cardiac management on AMI survival using propensity score and instrumental variable methods. JAMA. 2007;297:278-285.

25. Radford MJ, Foody JM. How do observational studies expand the evidence base for therapy? JAMA. 2001;286: 1228-1230.

26. Heney NM, Nocks BN, Daly JJ, et al. Ta and T1 bladder cancer: location, recurrence and progression. $\mathrm{Br} J$ Urol. 1982;54:152-157.

27. Stein JP, Lieskovsky G, Cote R, et al. Radical cystectomy in the treatment of invasive bladder cancer: long-term results in 1,054 patients. J Clin Oncol. 2001;19:666-675.

28. Anderson RT, Sorlie P, Backlund E, Johnson N, Kaplan GA. Mortality effects of community socioeconomic status. Epidemiology. 1997;8:42-47.

29. Manly JJ. Deconstructing race and ethnicity: implications for measurement of health outcomes. Med Care. 2006; 44(11 suppl 3):S10-S16. 